\title{
Hospital-based safety and tolerability study to assess efficacy of oral doxycycline in the treatment of Wuchereria bancrofti infection in north-eastern Tanzania
}

\author{
W.H. MAKUNDE ${ }^{1 *}$, L.M. KAMUGISHA ${ }^{1}$, R.A. MAKUNDE ${ }^{3}$, M.N. MALECELA ${ }^{2}$ \\ and A.Y. KITUA ${ }^{2}$ \\ ${ }^{1}$ National Institute for Medical Research, Tanga Research Centre, P. O. Box 950, Tanga, Tanzania \\ ${ }^{2}$ National Institute for Medical Research Headquarters, Dar es Salaam Tanzania \\ ${ }^{3}$ Bombo Regional Hospital, Tanga, Tanzania
}

\begin{abstract}
A hospital based open-label clinical trial of 19 apparently healthy adult males with microfilaraemia was conducted to assess safety, tolerability and efficacy of doxycycline on Wuchereria bancrofti. Study individuals were assigned 8 weeks treatment with doxycycline $200 \mathrm{mg}$ daily. The results of different selected tests showed that, the haematological, hepatic, renal and clinical parameters pre-and post-drug administrations were within the normal range for all treated individuals. Clinical adverse events were mild, transient, tolerable and reported in $7 / 19$ (36.8\%) of the study cohort. The mf clearance rate was $100 \%$ at 12 months post treatment for the 13 individuals who completed the follow up. These findings indicate that, although the drug was administered for a long period, there was no evidence of toxicity to the myocardium, hepatocytes, renal, bone marrow and blood cells, suggesting that an 8-week course of 200 $\mathrm{mg} /$ day doxycycline is a safe and tolerable regime for the treatment of Wuchereria bancrofti infections.
\end{abstract}

Key words: doxycycline, safety, efficacy, bancroftian filariasis, Tanzania

\section{Introduction}

Doxycycline is a broad-spectrum bacteriostatic antibiotic that came into clinical practice 22 years after the discovery of chlortetracycline, a member of the tetracycline group, in 1948. This drug is effective against gram-positive and gram-negative bacteria, rickettsiae, chlamydiae, mycoplasms and some gastrointestinal tract protozoa (http:// www.doxycycline.com). In recent years, the antirickettsial activity of doxycycline has been exploited as a novel treatment for filarial infections, targeting the endosymbionts rickettsiae-like bacteria, Wolbachia (Taylor et al., 2000, 2001; Hoerauf et al., 2000a). Doxycycline and other tetracyclines have shown promising antifilarial activity in human onchocerciasis and animal filarial parasite harbouring Wolbachia endobacteria (Hoerauf et al., 2000b; Townson et al., 2000).

Adverse events to doxycycline are infrequent and have similar clinical profile to fluoroquine and macrolide antibiotics. Although adverse effects are infrequent, precaution has to be taken when administering the drug to pregnant women, lactating mothers and children below 12 years of age. This is so because the drugs are selectively taken up in growing bones and teeth of foetus and children, a process believed to be due to the drug chelating properties with calcium phosphates. This process is believed to trigger dental enamel hypoplasia with pitting and cusp malformation (Kunin et al., 1959;
Storey \& Backer, 1970; Storey \& Basle, 1973; Wallman \& Hilton, 1962; Cohlan et al., 1963).

Exposure to sunlight for a long period on doxycycline or tetracycline has been associated with photo-toxicity reaction; onycholysis and pigmentation of the nails developing simultaneously at all ages (Horio, 1988). These reactions can occur when the skin is exposed to sunlight rays in the range of 270$320 \mathrm{~nm}$ (Carey, 1960). Although rarely, doxycycline can precipitate pre-existing renal failure. Studies have shown the drug to exacerbate existing renal failure in individuals with poor nutritional status and elderly people (Orr et al; 1978). This is attributed to the antianabolic effect of the drug. Like many other drugs, doxycycline has some gastro-intestinal tract tolerable undesired effects when used to treat bacterial infections. These adverse events are likely to be caused by the irritation of the gastric mucosa. It is for these reasons that, safety and tolerability of the doxycycline in the treatment of filarial diseases has to be established before adopting it for managing the diseases.

Although the drug is widely used in Tanzania for the treatment of malaria, cholera, plague, chronic bronchitis and sexually transmitted infections, there are no reports on hepatic and renal dysfunction or even photo-toxicity when normal oral doses are administered for any of the conditions mentioned earlier. Therefore, we carried out a hospital based open-label clinical trial to evaluate safety, tolerability and efficacy of doxycycline

*Correspondeonce: Dr. Williams Makunde; E-mail:hwmakunde@hotmail.com 
in local apparently 'healthy' adults with microfilariae of W.bancrofti.

\section{Material and Methods}

\section{Subjects}

Study individuals were males aged between 14 and 68 years old with microfilaraemia of at least $100 \mathrm{mf} /$ $\mathrm{ml}$; those who were resident in the study village for 5 years or more and had not taken antifilarial drugs, anthelmintics, doxycycline, tetracycline, rifampicin, chloramphenicol, phenobarbitone or amodiaquine during the previous six months. Individuals with a history of chronic liver, cardiac, renal or CNS disease, or allergy to doxycycline were excluded, as were those with hepatic and renal values outside the normal ranges (aspartate aminotransferase 0-40 IU/L, alanine aminotransferase 0-45 IU/L, creatinine 53-126 $\mu \mathrm{mol} /$ L). Similarly individuals with abnormal haematological indices were also excluded.

\section{Assessment of safety, tolerability and efficacy}

Doxycycline (Vibramycin ${ }^{\circledR}$, Pfizer) capsules were used in this study. Study individuals were weighed and received oral doses of $200 \mathrm{mg}, 2$ capsules of 100 $\mathrm{mg}$ each of the drug daily for eight consecutive weeks. The drug was administered at $10.00 \mathrm{~h}, 30$ minutes after breakfast of black tea, bread with mango jam to reduce or prevent gastric irritation. Treatment was supervised while dosing of the drug, an individual was observed for 20 minutes post drug administration. Study individuals were advised not to take anti-acids, milk or alcoholic beverages before taking the trial drug and during the entire treatment period.

Venous blood specimen was obtained through vein-puncture from the recruited individuals in $7 \mathrm{ml}$ EDTA tubes between $21.00 \mathrm{~h}$ and $24.00 \mathrm{~h} .6 \mathrm{ml}$ were used to process alanine aminotransferase (ALT), asparate aminotransferase (AST), creatinine, total bilirubin, haematological indices and circulating filarial antigen (CFA) by using immunochromatographic test (ICT) card (Trop-Ag ELISA kit TropBio, Townsville, Australia). $1 \mathrm{ml}$ of the specimen was diluted in water (1:4 ratios) and filtered through a $3 \mu \mathrm{m}$-pore-size membrane to determine microfilariae. These tests were carried out at day 0 pre-medication and 7 during treatment. However, at follow up, only $1 \mathrm{ml}$ of blood specimen was collected for assessing mff and circulating filarial antigenaemia from day 90 to day 360 post-treatment. Total white blood cell count was determined using Neubauer haematocytometer while a thin blood smear was prepared then stained with Giemsa and the type of white cell counted through a microscope out of $100 \%$. Haemoglobin was measured by Corning-Ell calorimeter with wavelength range of 400-700 $\mathrm{nm}$.
Clinical evaluation was undertaken at the hospital every 8 hours for the first 48 hours, then 12 hourly. Thereafter, monitoring was done daily for the 53 days at the community and the results were recorded on a clinical drug adverse event assessment form. The evaluation included assessment of blood pressure in supine, upright and sitting position, heart sound, axillary temperature, skin and eyes. Cardiac and respiratory rates and abdomen were also examined. Drug adverse reactions were recorded when study individuals reported them to the monitoring clinician as well as the directly observed events by the clinician. The observed clinical events were assessed using scoring method of $0=$ no adverse event, $1=$ mild event, $2=$ moderate event and $3=$ severe events. The scoring of 0 was when there was no event observed or reported and no alteration of normal daily task of the individuals was observed. Scoring of 1 was defined as mild adverse reaction with mild alteration whereby an individual was aware of and could easily tolerate and continue with daily activities; scoring of 2 was done when a moderate adverse event with moderate alteration which could cause discomfort and interference with daily activities was recorded; scoring of 3 was defined as a severe adverse event with severe alteration which could prevent usual daily activities and would require admission to hospital for treatment.

\section{Data analysis}

All data were entered and verified in Epi Info 6.04d and analysed using version 7 of the STATA software package (Stata Corp., College Station, TX). Data were analysed using non parametric (Wilcoxon Sign rank test) for continuous variable. Categorical data were analysed using $\chi^{2}$ tests and Fisher's exact test. Geometric mean intensity (GMI) of mff counts was calculated as antilog $\left[\sum(\log \mathrm{x}+1 / \mathrm{n})\right]$ with $\mathrm{x}$ being the number of $\mathrm{mf} / \mathrm{ml}$ of blood in microfilaraemic individuals and $n$ the number of microfilaraemic individuals examined. GMI in different individuals were compared by student's t-test and analysis of variance on log-transformed values.

\section{Ethical considerations}

This study ethical clearance approval of the Tanzania Medical Research Coordinating Committee the Liverpool School of Tropical Medicine, UK.

\section{Results}

A total of 20 individuals were recruited for the study. However, only 19 completed the 7-day safety and tolerability evaluation. Thirteen individuals were finally assessed for efficacy at 12 months post-drug administration. One subject was removed from the study after he was diagnosed to suffer from pulmonary 
tuberculosis. The pre-drug administration clinical examination parameters evaluated were all within normal range except two individuals whose total serum bilirubin (range $=0.4$ and $0.6 \mu \mathrm{mol} / \mathrm{L}$ ) raised above the normal values (range $=3.4-20.4 \mu \mathrm{mol} / \mathrm{L}$ ) and three individuals presented neutropenia of $36 \%, 39 \%$ and $38 \%$ were below the normal range (40-75\%).

Baseline, systolic-diastolic blood pressure of the subjects was within the normal limits (range 100-140 $\mathrm{mmHg}$ systolic; $60-90 \mathrm{mmHg}$ diastolic). The heart rate (64-88/min), respiration rate $(12-20 / \mathrm{min})$, and body temperature $\left(36.1-37.6^{\circ} \mathrm{C}\right)$ were within normal rates. At baseline all study individuals were positive for Wuchereria bancrofti infection without any clinical signs and/or symptoms of the disease.

Post doxycycline therapy, total white cell counts of all study individuals were all within normal range diarrhoea, epigastria pain and vomit. The most frequent and common drug adverse reaction observed was fever in 5/7 (71.4\%) with an intensity of 2 score out 5 episodes in terms of intensity. This adverse event occurred at day 2 post-drug administration. Vomiting in 4 candidates was reported on day 2 and 3 post-treatment. There was no individual who had to stop taking treatment because of adverse reactions.

At 6 month post-treatment, one individual, age 68 years, among those who reacted presented a dark-blue discolouration of his first and second right fingernail, which disappeared after two weeks. Those individuals who experienced adverse reaction, their mf GMI were higher at pre-treatment as compared to those who did not react. There was no significant difference in the mff status between individuals who experienced adverse events and those who did not $(P=0.2$; Mann Whitney test).

Table 1: The levels of microfilarial intensity pre-and post-treatment with doxycycline

\begin{tabular}{lcc}
\hline Days & Number of mff positive Individuals & Mff GMl/ml \\
\hline 0 & 19 & 461.7 \\
7 & 13 & 362.5 \\
90 & 10 & 168.2 \\
180 & 6 & 24.5 \\
270 & 2 & 4.0 \\
360 & 0 & 0 \\
\hline
\end{tabular}

(6.0-9.5 x 109/L). However $15.8 \%(3 / 19)$ of the subjects presented relative lymphocytosis of $10 \%$, $11 \%$ and $3 \%$ (normal range $20-40 \%$ ). There were three individuals who presented eosinophilia, with an increase of $3 \%, 9 \%$ and $2 \%$ above the normal range (1-3\%). Haemoglobin levels and thrombocytes count were within the normal range for individuals living in the lowland coast of Tanzania. Similarly biochemical functional tests including AST (10-40 IU), ALT (0-45 IU, total serum bilirubin (3.4-20.4 $\mu \mathrm{mol} / \mathrm{L})$ and creatinine $(53-126 \mu \mathrm{mol} / \mathrm{L})]$ were within the normal ranges. Clinical parameters evaluated at post-drug administration (blood pressure, heart rate, respiratory rate, body temperature and weight) were within the normal range.

Seven individuals (36.8\%) experienced one or more signs or symptoms that were associated with posttreatment adverse drug reactions in the preceding seven days. All drug adverse events occurred on day 2 after start of treatment. The adverse reactions reported after doxycycline administrations were mild and tolerable. Of the individuals who experienced adverse reactions, the majority (5/7) reported more than one symptom. The adverse reactions were characterised by fever, headache,
Six individuals could not keep to the examination schedule as it interfered with their fishing activities. They were both clinically normal and their laboratory tests were within the normal range at the time they dropout to the follow up at three month. The geometric mean density of microfilaraemia at different days post-therapy was expressed as a percentage of the pretreatment geometric of the positive individuals. By day 7 post-treatment, the mff geometric reduction intensity was $21.5 \%(99.2 / 461.7)$ of the pre-treatment mff levels (Table 1). However, by the third month post-treatment the mff levels had decreased to $36.4 \%$ (168.2/461.7). A high proportion of reduction in the intensity of infection was observed between the $6^{\text {th }}$ and $9^{\text {th }}$ month post-treatment with mff GMI pretreatment ranging from $63.6 \%$ to $94.7 \%$. At 12 moths post treatment mff GMI was reduced by $100.0 \%$ $(P<0.05)$. ICT for circulating antigenaemia in the study cohort showed $100 \%$ antigen-positive reactions at pre-treatment. However, at 9 and 12 months posttreatment, the intensity of ICT reaction was weak (reduced intensity towards negative) in two of the study cohort. 


\section{Discussion}

During the entire period of safety monitoring of the study subjects, only mild tolerable adverse reactions attributable to doxycycline were observed. These effects did not require admission at the hospital unit for special care. Clinically, the vital signs were within normal range during treatment and post-treatment periods. Similarly, there were no marked changes in the biochemical functional values and haematological parameters. In general, there were no serious allergic toxic reactions associated to doxycycline in our study subjects. These results have therefore provided a wide safety scope of the drug. These findings correlate with other studies, which showed the rarity of adverse events triggered by doxycycline (Bigby, 1986; Raeder, 1984).

The discolouration of nails observed in one of the study candidates after eight weeks of oral taking of the drug is not an alarming event. Similar reaction has been observed in subjects taking tetracycline for even a short course when exposed to direct sunlight (Carey, 1960; Horio, 1988; Bjellerup \& Ljunggren, 1994). Although the direct sunlight toxic effects have been experienced in white skin races, it is not clear whether this type of reaction is common among black skin races. It is likely that, the affected candidate was more exposed to direct sunlight due to his fishing or farming activities during the day. The main activity of the study population is farming which usually, does not expose them to direct sunlight because they are working under the tree shade. It is also important to note that photosensitivity reactions are adverse cutaneous responses to the combined action of drug and a physical agent (light) (Harber \& Bickers, 1989). The onset of these reactions has been observed to occur minutes to hours after drug administration and peaks a few hours to several days later. It was difficult to predict when the reaction occurred in our patient, as he could not remember when exactly the reaction started.

Our findings indicated that all the neutropenia at pre-treatment resolved to normal values at posttreatment. One candidate had his lymphocytosis level reduced by $1 \%$ (54\% at pre-treatment to $53 \%$ at posttreatment). On the other hand, subjects with eosinophilia at pre-treatment had resolved to normality at post-treatment and new cases were a noted with increases of 3\%, 9\% and 2\%. Eosinophilia both pre and post treatment are mostly likely to reflect to an on-going helminth infection and are unlikely to be a response to the drug.
The frequency of treatment related clinical adverse events were comparable between individuals. Moderate to severe headache has been reported in tetracycline treatment of infants and children because the drug induced pseudotumor cerebri leading to intracranial hypertension (Olson, 1966). In our case, it is unlikely that the cause of headache in our study subjects was intracranial hypertension because children were not among our study cohort. The adverse reactions experienced in our study subjects do not differ with those experienced in antifilarial drugs (Addis, 1997; Dreyer et al., 1994; Kumaraswami et al., 1988).

Although frequent serious oesophageal ulcerations has been associated with doxycycline (Hatheway, 1982; Shiff, 1986) none of our study cohort presented/complained of this type of reaction. The incidence rates and intensity of gastrointestinal intolerance may be an important aspect to be monitored if the drug has to be adopted for filarial chemotherapy. Since doxycycline absorption is not affected by food in the upper gastrointestinal tract segment, therefore small amount of meals can be given to reduce gastric irritation (Quick et al., 2002). Gastrointestinal tract adverse reactions (nausea, vomit, abdominal pain and anorexia), are not uncommon phenomena in many drugs including the antibiotics, therefore, one should anticipate such reactions when treating patients with the drug. Ansonia, rash, itching, jaundice, atypical lymphocytosis and thrombopenic purpuras (Carey, 1960) are some of the rare unwanted effects associated with administration of the drug. These serious adverse events were not experienced in our study subjects.

There were no significant clinical changes in the functional renal and hepatic biochemical enzymes activities. Our safety results are comparable with those reported for a six-week course of doxycycline in individuals with bancroftian filariasis in Ghana, although the infection intensity of the Ghana study was lower as compared to this present study (Hoerauf et al., 2003). Despite the long treatment period there was no alteration of hepatocytes, myocardium and skeleton muscles enzymes activity, indicating that the drug did not induce necrosis/damage to the liver cells or myocardium. The elevation of total serum bilirubin in one individual at pre-treatment is likely to be associated with the prolonged period without taking meals especially when these individuals are away from home for fishing trips. Since fasting is known to increase serum levels of bilirubin, it is likely that the prolonged 12-24 hours fasting could have contributed to the raised total serum bilirubin. 
The observed neutropenia is a common observation in Africans adults due to relative lymphocytosis or asymptomatic viral, bacterial or parasitic infections, which were not detected during study period. Our study results are consistent with adverse effects that are commonly seen when the drug is administered for bacterial and parasitic infections in routine practice, demonstrating that, doxycycline administered at this dose for 8 weeks does not induce serious adverse reactions. Moreover, the reduction of microfilaraemia to low levels 6 to 9 months posttreatment, and to amicrofilaraemic at the end of the study, indicate clearly that doxycycline is effective in the treatment of bancroftian filariasis.

It is suggested here that, future studies using shorter regimes of doxycycline alone or incombination with antifilarial drugs (e.g. diethyl carbamazine) already known to have adulticidal activity (Noroes et al., 1997; Eberhard et al., 1997; Dreyer et al., 1995) should be encouraged. Such studies should also incorporate more women as well as monitor the rates of development of drug resistance. It has already been reported in Tanzania such drug resistance was observed following an excessive use during cholera outbreaks in 1978 (Mhalu et al., 1979). In conclusion, although doxycycline was administered for a prolonged period of eight weeks, it did not induce serious adverse reactions. The drug is safe, tolerable and demonstrated suppression of W.bancrofti microfilariae, supporting its application in the treatment of filarial infection.

\section{References}

Addis, D.G., Beach, M.J., Streit, T.G., Lutwick Suzanne. L., Leconte, F.H., Lafontant, J.G., Hightower, A.W. \& Lammie, P.J. (1997) Randomised placebo-controlled comparison of ivermectin and albendazole alone and in combination for Wuchereria bancrofti microfilaraemia in Haitian children. Lancet 350, 480-484.

Bigby, M. (1986) Allergic reactions attributed to doxycycline among recipients of the drug. Journal of American Medical Association 256, 3358.

Bjellerup, M. \& Ljunggren, B. (1985) Photohaemolytic potency of tetracyclines. Journal of Investigative Dermatology 84, 262264.

Carey, B.W. (1960) Photodynamic response of a new tetracycline. Journal of the American Medical Association 172, 1196.
Cohlan, S.Q., Beveland, G. \& Tiamsic, T. (1963) Growth inhibition of premature receiving tetracycline. Clinical and laboratory investigation. American Journal of Disease in Children 105, 453-461.

Dreyer, G., Amiral, F., Norıes, J., Medeiros, Z. \& Addiss, D. (1995) A new tool to assess the adulticidal efficacy in vivo of antifilarial drugs for bancroftian filariasis. Transactions of the Royal Society of Tropical Medicine Hygiene 89, 225-226.

Dreyer, G., Pires, M.L., Andrade, L.D., Lopes, E., Medeiros, Z., Tenorio, J., Coutinho, A., Noroes, J. \& Figueredo-Silva, J. (1994) Tolerance of diethylcarbamazine by microfilaraemic and amicrofilaraemic individuals in an endemic area of bancroftian filariasis, Recife, Brazil. American Journal of Tropical Medicine and Hygiene 88, 232-236.

Eberhard, M.L., Hightower, A.W., Addiss, D.G. \& Lammie, P.J. (1997) Clearance of Wuchereria bancrofti antigen after treatment with diethylcarbamazine or ivermectin. American Journal of Tropical Medicine and Hygiene 57, 483- 486.

Harber, L.C. \& Bickers, D.R. (1989) Drug Induced Photosensitivity (Phototoxic and Photoallergic Drug Reactions). In: Harber, L.C. \& Backers, D.R. (eds). Photosensitivity Diseases. Principles of Diagnosis and Treatment. $2^{\text {nd }}$ edn. Toronto, BC: Decker Inc; Pp.160-202.

Hatheway, G.J. (1982) Doxycycline-induced esophagitis. Drug Intelligence \& Clinical Pharmacology 16, 879-880.

Hoerauf, A., Mand, S., Volkmann, L., B,ttner, M., Marfo-Debrekyei, Y., Taylor, M., Adjei, O. \& Buttner, D.W. (2003) Doxycycline in the treatment of human onchocerciasis: kinetics of Wolbachia endobacteria reduction and inhibition of embryogenesis in female Onchocerca worms. Microbes Infection 5, 261-273.

Hoerauf, A., Volkmann, L., Hamelmann, C., Adjei, O., Autenrieth, I.B., Fleischer, B. \& B, ttner, D.W. (2000a) Endosymbiotic bacteria in worms as targets for a novel chemotherapy in filariasis. Lancet 8, 1242-1243.

Hoerauf, A., Volkmann, L., Nissen-Paehle, K., Schmetz, C., Autenrieth, I., Buttner, D.W. \& Fleischer B (2000b) Targeting of Wolbachia endobacteria in Litomosoides sigmodontis: comparison of tetracycline with chloramphenicol, 
macrolides and ciprofloxacin. Tropical Medicine and International Health 5, 275-279.

Horio, T. (1988) Spontaneous photo-anycholysis. Journal of Dermatology 6, 540-542.

Kumaraswami, V., Ottesen, E.A., Vijayasekharan, V., Umadevi, S., Swaminathan, M., Aziz, M.A., Sarma, G.R., Prabhakar, R. \& Tripathy, S.P. (1988) Ivermectin for the treatment of Wuchereria bancrofti filariasis: efficacy and adverse reactions. Journal of American Medical Association 259, 3150-3153.

Kunin, C.M., Dornbush, A.C. \& Finland, M. (1959) Distribution of four tetracycline analogues in young men. Journal of Clinical Investigation 38, 1950-1963.

Mhalu, F.S., Mmari, P.W. \& Ijumba, J. (1979) Rapid emergence of El Tor Vibrio cholerae resistance to antimicrobial agents during the first six months of fourth cholera epidemic in Tanzania. Lancet 1 (8112), 345-347.

Norões, J., Dreyer, G., Santos, A., Mendes, V.G., Medeiros, Z., Coutinho, A. \& Addiss, D. (1997) Assessment of the efficacy of diethylcarbamazine on adult Wuchereria bancrofti in-vivo. Transactions of the Royal Society of Tropical Medicine and Hygiene 91, 78-81.

Olson, C.A. \& Riley, H.D. Jr. (1966) Complication of tetracycline therapy. Journal of Paedriatics 68, 783-791.

Orr, L.H.Jr., Rudisill, E.Jr., Brodkin, R. \& Hamilton, R.W. (1978) Exacerbation of renal failure associated with doxycycline. Archives of Internal Medicine 138, 793-794.
Quick, J., Hogerzeil, H.V., Velasquez, G. \& Rago, L. (2002) Twenty-five years of essential medicine. Bulletin of the World Health Organization 80, 913-914.

Raeder, J.C. (1984) Anaphylactoid reactions due to doxycycline. Drug Intelligence \& Clinical Pharmacology 18, 481.

Shiff, J. (1986) Oesophageal ulceration. A Geschwind Medical Journal of Australia 256, 1893.

Storey, E. \& Backer, K.L. (1970) Administration of tetracycline in the first 3 years of life was associated with high risk of permanent molar damage. Medical Journal of Australia 1, 109.

Storey, E. \& Basle, D.(1973) A brief review of the discoloration of the teeth of children given tetracycline. Medical Journal of Australia 6,321.

Taylor, M.J., Bandi, C., Hoerauf, A.M. \& Lazdins, J. (2000) Wolbachia bacteria of filarial nematodes: a target for control. Parasitology Today 16, 179180.

Taylor, M.J., Cross, H.F., Ford, L., Makunde, W.H., Prasad, G.B. \& Bilo, K. (2001) Wolbachia bacteria in filarial immunity and disease. Parasite Immunology 23, 401-409.

Townson, S., Hutton, D., Siemienska, J., Hollick, L., Scalon, T., Tagboto, S.K. \& Taylor, M.J. (2000) Antibiotics and Wolbachia in filarial nematodes: antifilarial activity of rifampicin, oxytetracycline and chloramphenicol against Onchocerca gutturosa, Onchocerca lienalis and Brugia pahangi. Annals of Tropical Medicine and Parasitology 94, 801-816.

Wallman, I.S. \& Hilton, H.B. (1962) Teeth pigmentation by tetracycline. Lancet, 1, 827-829. 